\title{
A CFD Benchmark of Active Flow Control for Buffet Prevention
}

\author{
Fulvio Sartor \\ ONERA - Applied Aerodynamics Department. Meudon, France \\ fulvio.sartor@onera.fr \\ Mauro Minervino \\ CIRA - Fluid Mechanics Department. Capua, Italy \\ m.minervino@cira.it \\ Jochen Wild \\ DLR - Institute of Aerodynamics and Flow Technology. Braunschweig, Germany \\ jochen.wild@dlr.de \\ Stefan Wallin \\ KTH - FLOW Centre, Dept. of Mechanics. Stockholm, Sweden \\ stefanw@mech.kth.se \\ Hans Maseland \\ NLR - Netherlands Aerospace Centre. Amsterdam, The Netherlands \\ hans.maseland@nlr.nl \\ Julien Dandois \\ ONERA - Applied Aerodynamics Department. Meudon, France \\ julien.dandois@onera.fr \\ Vitaly Soudakov \\ TsAGI - Department of Aerodynamics. Zhukovsky, Moscow Region, Russia \\ vit_soudakov@tsagi.ru \\ Petr Vrchota \\ VZLU - Aeronautical Research and Test Institute. Prague, Czech Republic \\ vrchota@vzlu.cz
}

\begin{abstract}
This paper will present the main results of the aerodynamic design and analysis for flow control applied to trailing edge of wings and profiles. This work has been conducted in the framework of the European project AFLoNext aiming at developing technologies allowing for an improvement of the performance and loads situation in the operational domain. The technologies are expected to provide an increase in aerodynamic efficiency and a structural weight reduction for the design flight conditions with a potential for 1-2\% fuel savings and corresponding emission reduction. Numerical simulations are performed on 2D and 3D test cases. Where available, a comparison with experimental data is performed. High-speed flow is considered, in order to investigate a transonic configuration representative of cruise conditions. Trailing edge devices (TED) such as fluidic Gurney flaps or micro-jets for circulation control are used for assessing the possibility of delaying the buffet onset or increasing the maximum achievable lift, thus extending the flight envelope of an aircraft. The purpose of the present paper is to present the result of the work performed by the different partners involved in the project.
\end{abstract}

\section{KEYWORDS}

RANS, transonic buffet, flow control, Trailing Edge Device 


\section{NOMENCLATURE}

$\begin{array}{ll}\alpha & \text { angle of attack } \\ \text { CFD } & \text { Computational Fluid Dynamics } \\ C_{D} & \text { Drag Coefficient } \\ \mathrm{Pi}_{T E D} & \text { Total pressure of the TED }\end{array}$

$\begin{array}{ll}c & \text { chord of the profile } \\ C_{p} & \text { Pressure Coefficient } \\ C_{L} & \text { Lift Coefficient } \\ \text { TED } & \text { Trailing Edge Device }\end{array}$

\section{INTRODUCTION}

The current growth of air traffic worldwide (expected to double every fifteen years) emphasizes clearly the stakes of fuel-burn reduction, both from an environmental and economical perspective. The operating costs of transport aircraft are largely affected by the aerodynamic performance of the wing. Because of rising fuel costs and lower emissions targets there is an increasing need to exploit the full aerodynamic potential of the wing, either across the entire operating envelope, or at design flight conditions. In the past, the ability to modify the detailed flow at the trailing edge of a wing has been shown to potentially provide aerodynamic benefits by and adaptive change of camber during flight and to improve aircraft performance across the entire flight envelope.

A number of trailing edge devices for the control of trailing edge flows have been proposed: these include a variety of "Gurney" flaps $[12,2,6]$ or "Mini-Trailing Edge Device" [18]. More recently, the use of fluidic injection at the trailing edge of a wing has been suggested [11, 10] and, to a limited extent, evaluated both theoretically and experimentally [3]. These fluidic devices include concepts such as normally blown fluidic Gurney flaps and tangentially blown "Circulation Control" concepts [8]. The basic idea is to replace the mechanical trailing edge deflector, which has shown to be able to increase the wing aerodynamic performances and delay the onset of buffet by altering the load distribution [3], using a fluidic devices.

In the case of transonic buffet phenomenon, it has been shown that fluidic vortex generators located upstream of the shock foot can reduce the extent of the separated area and are very efficient to postpone the buffet onset [22]. However, the effect of the fluidic TED is different, the separation is not suppressed, but the rear wing loading is increased and consequently the buffet onset is not delayed to higher angles of attack, but only to higher lift coefficient [13]. Fluidic TED have also found applications on nozzle engines: fluidically enhanced chevrons have also shown promising results for supersonic jet noise reduction [20].

One of the goals of the AFLoNext project is to explore the potential of active mechanical and pneumatic trailing edge flow control concepts for application in a multi-function role at both high- and low-speed operating conditions. This is done by application of numerical simulation together with targeted simple experiments to understand the potential aerodynamic performance benefits achievable together with design parameters such as pneumatic supply pressures/mass-flows and the required speeds/frequencies of operation.

The partners involved in the project focussed on the numerical assessment of the flow-control devices and their effect on a reference configuration. The numerical simulation is a central part of this collaborative project, and is done to understand both the flow inside a control device and the impact on the total aerodynamic field in each considered case. Parametric investigation is used to assess the effect of the control-device location on the global system, while optimisation is applied in order to assist the design of the actuator and define its geometric characteristics. Different goal functions have been considered for the optimisation process, aiming at maximising the total lift, the aerodynamic efficiency, or at obtaining an increase of the lift without incurring in buffet conditions. The design variables were the jet orientation, the slot width, its position, and the stagnation pressure intensity. In order to investigate the multi-functional use of the TED, a low-speed application was also considered on a high-lift aerofoil equipped with a simple single-slotted trailing-edge flap with TED.

The paper is structured as follows: first, the numerical benchmark is presented in section 2, where the partners evaluated the performance of their code by comparing RANS results on cases with and without flow control against an existing experimental database. The results are then presented: first in section 3 in the case of a 2D profile with TED, then in section 4 for a half wing-body configuration equipped with a 3D slot acting as a fluidic Gurney flap on the outer part of the wing. 


\section{NUMERICAL BENCHMARKS}

The design of an efficient control device needs an accurate description of the aerodynamic field of the uncontrolled configuration as well as a correct representation of the control device itself. The work performed in this article is based on numerical simulations. A validation process is necessary to assess the capability of modern CFD techniques of correctly predicting the behaviour of the flow in the presence of control devices. For this reason, two numerical benchmarks have been proposed and a comparison with available experimental data allows for code validation. Different codes are used by the partners involved in the simulations, for example U-ZEN by CIRA [1], elsA by ONERA [19], TAU by DLR [5], Edge by KTH and VZLU[21]. The results by KTH has been obtained in cooperation with FOI, the Swedish Defence Research Agency.

The benchmarks focus on a 2D profile and a 3D half wing-body configuration in transonic conditions, with and without control device at the trailing edge. When the angle of attack is small, the interaction between the shock and the boundary layer yields to a steady flow field. When increasing the angle of attack, periodic shock motions known as transonic buffet occur, and the flow is unsteady. The experimental results are available from the European FP6 project AVERT, were two wind tunnel testing campaigns have been performed, aiming at studying buffet and delaying its onset by means of several control devices. The first one was performed at VZLU A4 test section wind tunnel with an existing ONERA 2D OAT15A aerofoil [14]. The second test campaign was performed at the ONERA S2MA wind tunnel on a $3 \mathrm{D}$ half-model $[15,16]$.

\section{RESULTS OF THE 2D TEST CASE}

The aerofoil geometry is based on a modified ONERA OAT15A cross-section. The chord length and span of the used wind tunnel model are equal to $0.200 \mathrm{~m}$ and $0.390 \mathrm{~m}$, respectively. This model, visible in figure 1, is equipped with a removable mechanical TED that can be easily modified and thus has been adapted to integrate a fluidic TED. Moreover, its wing span was compatible with the dimensions of the VZLU-A4 test section with a few adjustments. This model was tested at the transonic ONERA T2 wind tunnel in two campaigns in 1998 and 1999 [14].

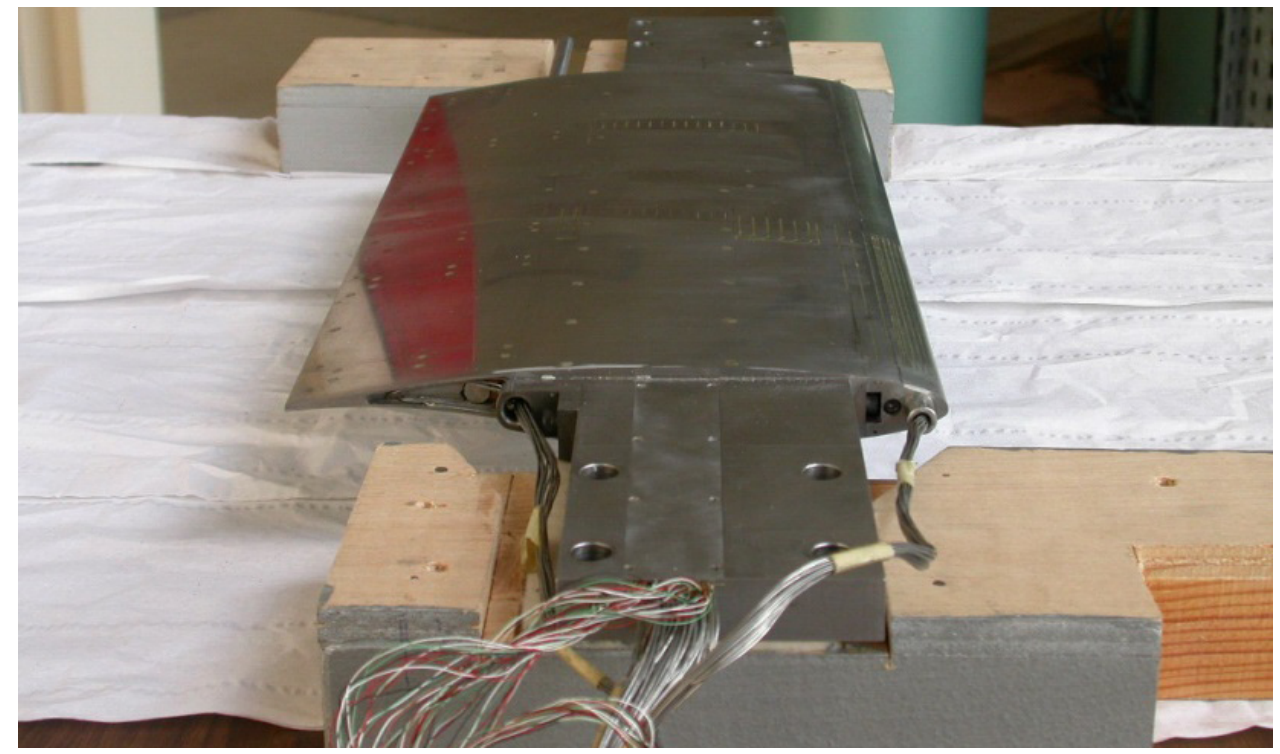

Figure 1: OAT15A aerofoil for 2D buffet characterisation tested in the VZLU A4 wind tunnel.

The test conditions were a variable Mach number between 0.730 and 0.739 , stagnation pressure of 1 bar and a Reynolds number based on the chord of 2.6 million. The transition was fixed at $7 \%$ of chord length on both upper and lower side. The main part of the test program was related to the 
aerodynamic evaluation of the reference configuration (reference standard trailing edge, and/or baseline configuration with fluidic TED without blowing) and, above all, aerodynamic evaluation of fluidic TED with blowing. Static pressure (pressure taps) and unsteady pressure (Kulite transducers) measurements were performed, while some specific oil-flow visualizations allowed to check side walls interferences. For the baseline configuration, the buffet characteristics (onset, shock position and intensity) obtained at the VZLU A4 transonic wind tunnel are very consistent with the former ones recorded at the ONERA T2 transonic wind tunnel testing campaigns, allowing then to pursue on fluidic TED investigations.

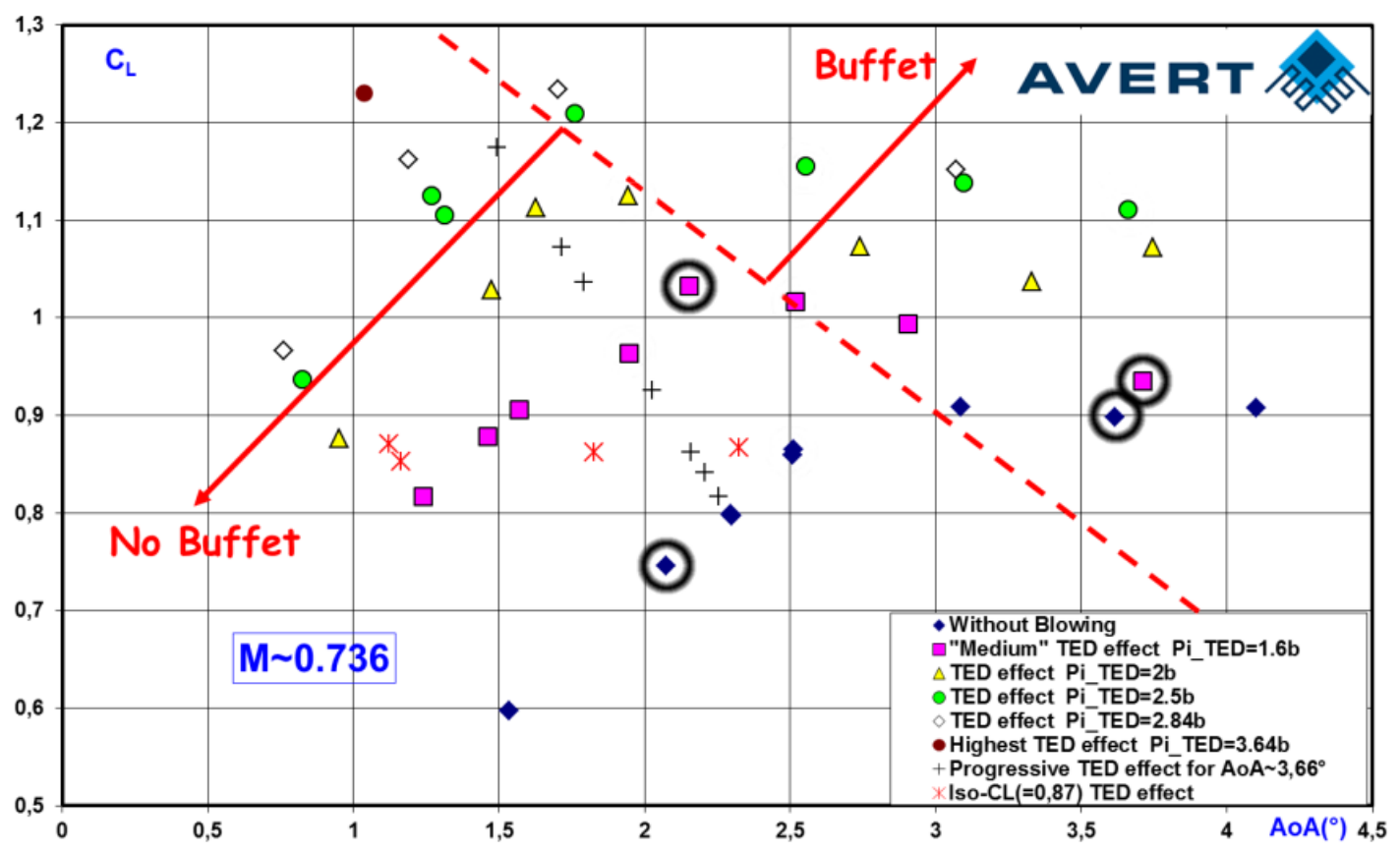

Figure 2: Polar curves with the selected test cases for the 2D benchmark exercise.

Nine test cases are proposed before and after buffet onset, for the baseline configuration and for two levels of TED mass flow rate. They can be clearly identified (rounded by black circles) in figure 2. The following angles of attack will be presented in this paper:

- Mach $=0.7439, \alpha=2.03^{\circ}$ (no buffet), without blowing

- Mach $=0.7385, \alpha=3.58^{\circ}$ (buffet), without blowing

- Mach $=0.7316, \alpha=2.02^{\circ}$ (no buffet) blowing at $\mathrm{Pi}_{T E D}=1.6$

- Mach $=0.7366, \alpha=3.71^{\circ}$ (buffet), blowing at $\operatorname{Pi}_{T E D}=1.6$

The partners were provided with the geometrical characteristics of the 2D aerofoil and the slot, and for each test case with the lift coefficient (obtained by pressure integration) and chord-wise distributions of $C_{p}$ and $C_{p}$ RMS. To simulate the different test cases all the partners generated a multi-block structured C-type hexahedral mesh appropriately refined in the proximity of the TED slot and around the expected shock location. The flow control device was either simulated using a surface boundary condition on the profile, which imposes the stagnation pressure and temperature at the TED slot outlet, (CIRA, TsAGI, VZLU), or with total pressure and temperature imposed at the inlet of an idealized slot geometry (DLR, ONERA), or with total pressure and temperature imposed at the inlet of the provided slot geometry (ONERA). The three approaches are summarised in figure 3. Almost identical results were obtained for the first two modelling techniques, while the ONERA slot geometry yielded to an under estimation of the flow control effect. 

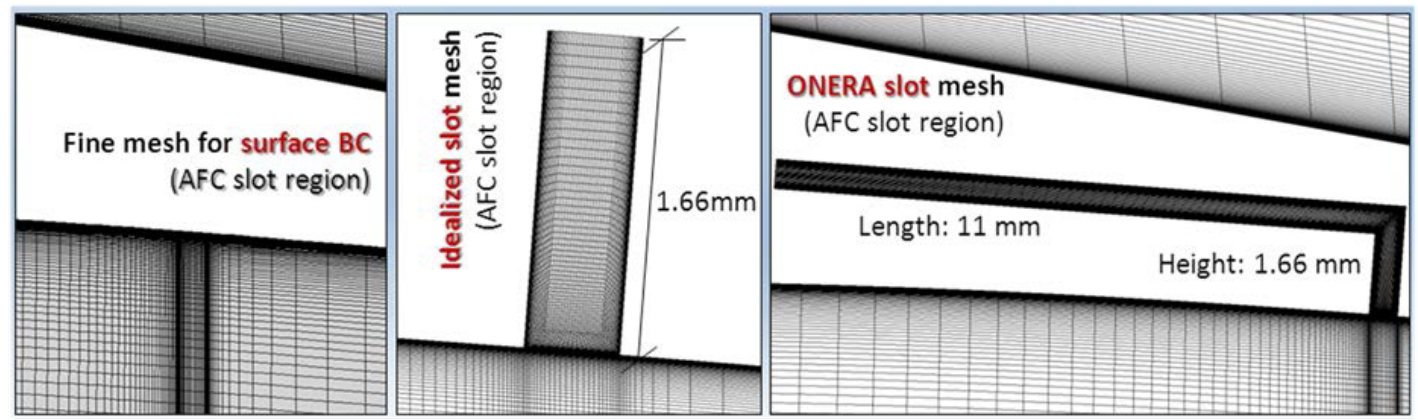

Figure 3: Different strategies for TED representation: surface boundary condition (left), idealized slot geometry (middle) or ONERA slot geometry (right).

Several turbulence models were considered, with most of the partners preferring the Menter k- $\omega$ SST model or the Spalart-Allmaras model. The spatial discretisation was based upon a finite volume formulation, with second and fourth order artificial dissipation.

Figure 4 shows on the left the pressure and skin friction coefficient distributions for the case at low angle of attack without blowing. The $C_{p}$ exhibits a very good agreement between all partner's results on the plateau level as well as on the shock position and the compression downstream of the shock. The shock is just located a bit too far downstream ( $2 \%$ of chord) in the CIRA computation. The discrepancies between partner's results are higher on the skin friction coefficient, presented on the right side of figure 4 , but the agreement is still reasonable. These larger differences can be explained by the fact that the skin friction coefficient is sensitive not only on the turbulence model, but also to the numerical approach implemented in each code.
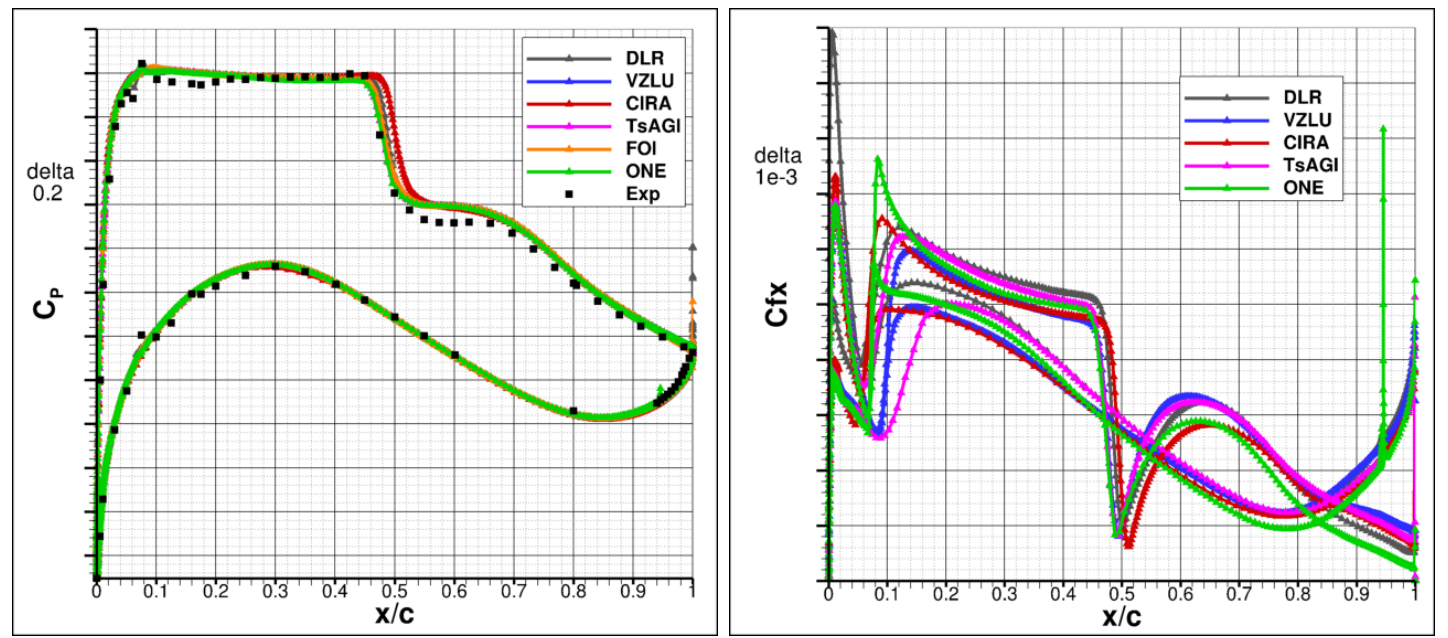

Figure 4: Pressure (left) and skin-friction (right) coefficients: low angle of attack, without TED.

The second test case is in the buffet regime and corresponds to a Mach number equal to 0.7385 and and $3.58^{\circ}$ angle of attack. Figure 5 shows on the left the time-averaged wall pressure coefficient. Much effort has been put in looking for modified flow conditions, in order to take into the account wind-tunnel corrections. CIRA computed both the original and modified flow conditions with a lower angle of attack in order to correct the wall effect in the wind tunnel. This new angle of attack should correspond better to the actual flow conditions seen by the model in the wind tunnel. The shock is located too far downstream for both CIRA and TsAGI and the plateau level is a little bit too high. The ONERA computation is in better agreement with the experimental data. The RMS values of the wall pressure coefficient are shown on the right side of figure 5 . There is a problem in the experimental data for the three sensors located 
between $40 \%$ and $45 \%$ of aerofoil chord, since there should be a peak like in the computations. There is also a problem on the position of the peak which seems more upstream in the experimental data than in the computations although there is a good agreement on the shock location.
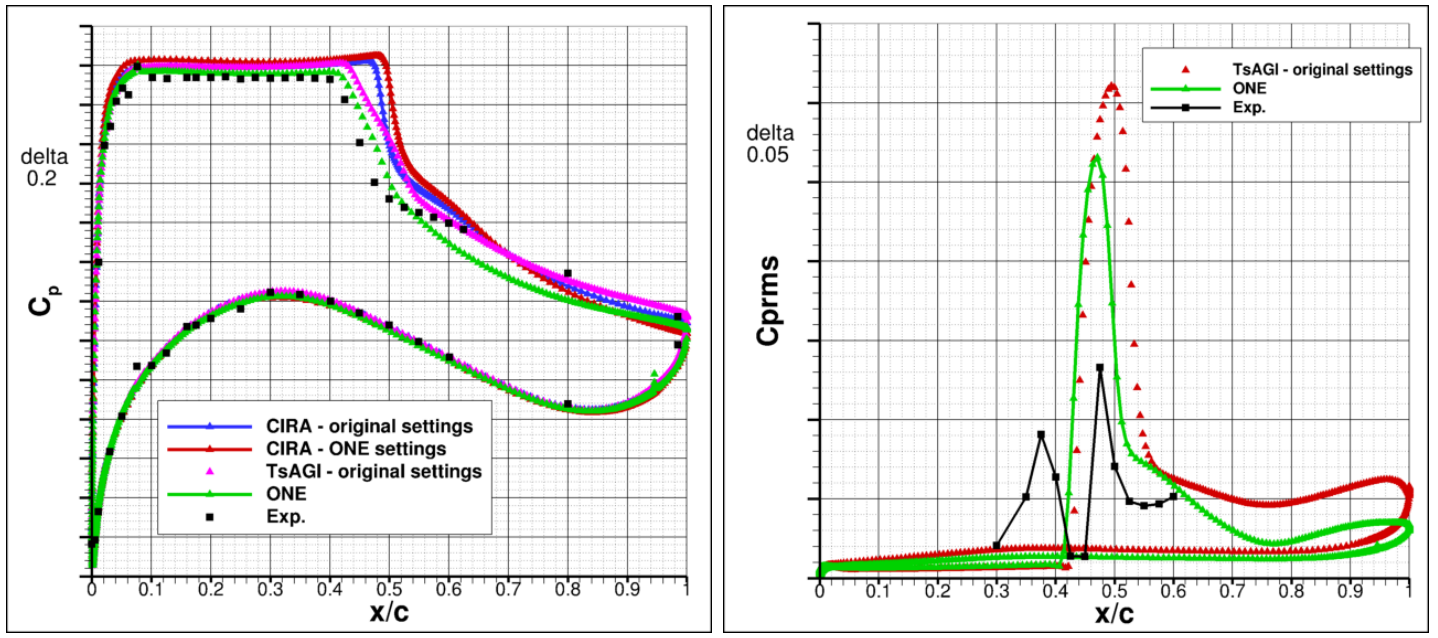

Figure 5: Mean (left) and RMS (right) values of pressure coefficient: high angle of attack, without TED.

The first case with the control device blowing is presented on the left side of figure 6 . The flow conditions are a Mach number of 0.7316 and $2.02^{\circ}$ angle of attack. ONERA performed two computations: one for which the flow is computed in the full cavity of the TED and the second one for which only the flow in the slot is computed like for DLR. In the VZLU, CIRA and TsAGI, the blowing boundary condition is applied at the wall, so the flow in the blowing slot is not computed. The pressure coefficient distribution shows a good agreement between all partner's results on the plateau level as well as on the shock location. The ONERA computation with the full cavity predicts a shock which is too upstream compared to the ONERA computation with just the slot and all the other computations too. This is due a separation in the $90^{\circ}$ corner in the cavity. On the contrary, for the simulations with just the slot (ONERA, DLR) or the boundary condition applied directly on the wall (VZLU, CIRA and TsAGI), the velocity profile has a top-hat shape and the numerical simulations are in better agreement with the experimental data. The best agreement on the wall pressure on the pressure side between $x / c=95 \%$ and $100 \%$ is obtained for the DLR and ONERA computation with the simplified slot. This region corresponds to the separated zone downstream of the fluidic TED.

The last case is in the buffet regime with the fluidic TED blowing, the original flow conditions were Mach equals 0.7366 and $3.71^{\circ}$ angle of attack. ONERA proposed a corrected condition with same Mach number but lower angle of attack. The left side of figure 7 shows that VZLU, TsAGI and ONERA computations predict a shock oscillation as expected but not the CIRA one. The time-averaged shock location is well predicted by TsAGI and ONERA computations and is a slightly too far downstream and with a lower amplitude for the VZLU one. Concerning the $C_{p}$ RMS distribution, presented on the right side of figure 7, the TsAGI and ONERA computations predicted a satisfying agreement in terms of $C_{p}$ RMS peak amplitude but this peak is located slightly too far downstream, which is surprising because the time-averaged shock location was well predicted on the $C_{p}$ distribution.

Globally, a good agreement between each partner's results and the experimental data has been observed on the wall pressure distribution as well as on the plateau level and the shock location. There are more discrepancies on the skin friction distribution and for the unsteady cases in buffet regime which are more challenging. Concerning the modelisation of the fluidic TED, different solutions have been chosen. Applying an injection boundary condition at the wall leads to a good prediction of the plateau level and shock location but the pressure distribution downstream of the fluidic TED is not very well predicted. Computing the flow in the full cavity, even if it seems more realistic compared with the wind tunnel tests, leads to the prediction of a shock which is too upstream. Computing the flow only in the slot leads to an overall better prediction as well as on the shock location and the pressure distribution downstream 

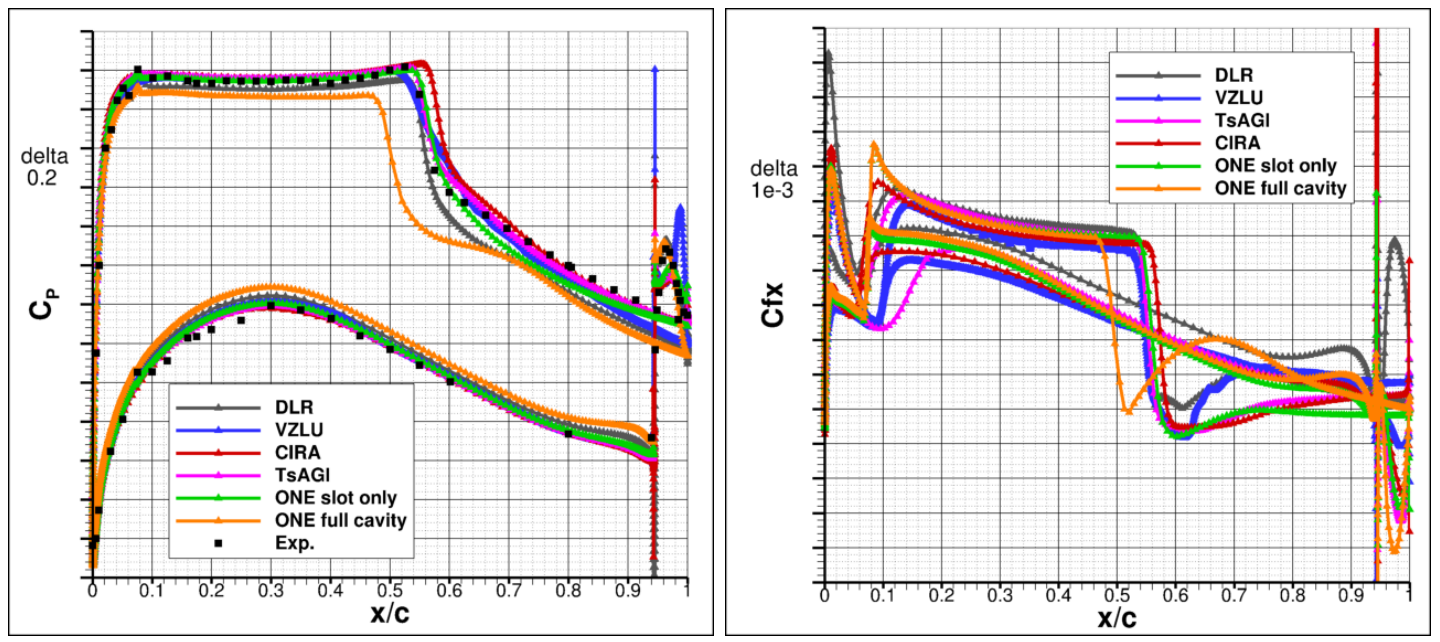

Figure 6: Pressure (left) and skin-friction (right) coefficients: low angle of attack, with TED.
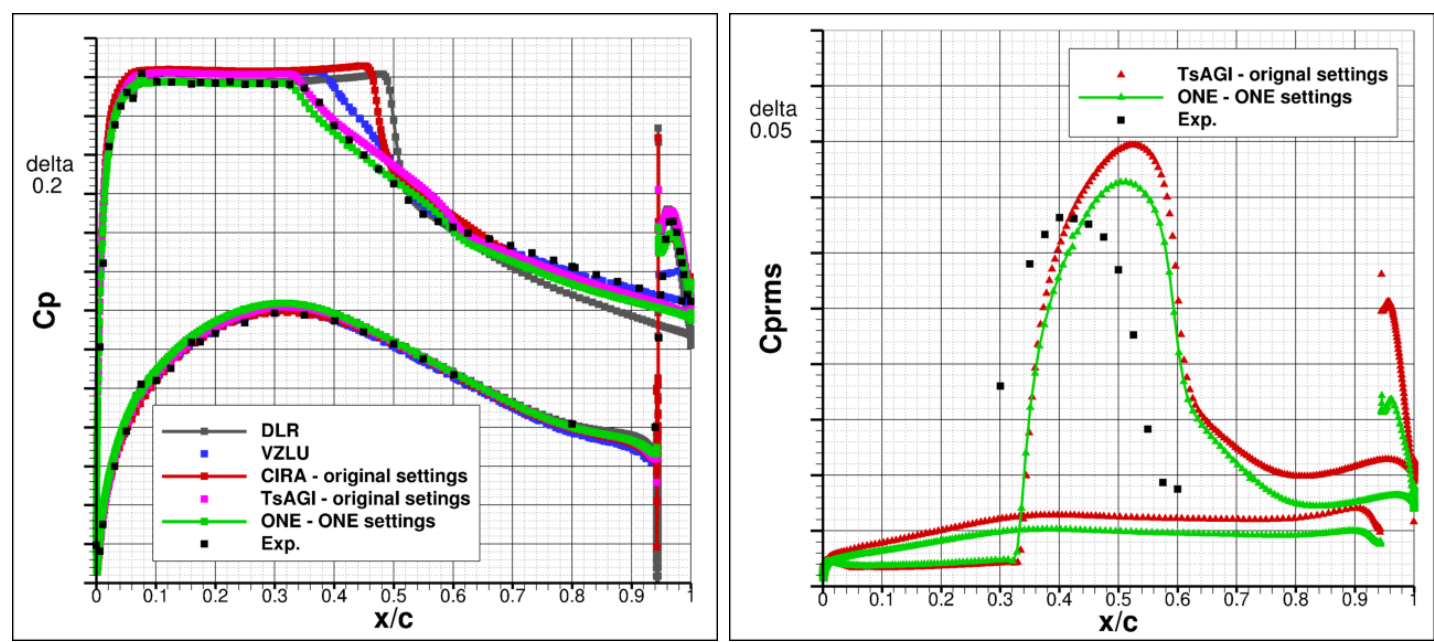

Figure 7: Mean (left) and RMS (right) values of pressure coefficient: high angle of attack, with TED.

of the fluidic TED. Concerning the prediction of the buffet onset, this onset is delayed to a higher angle of attack $\left(0.5^{\circ}\right.$ to $\left.1^{\circ}\right)$ than in the experimental data. The angle of attack correction depends on the turbulence model which is used.

\section{RESULTS OF THE 3D TEST CASE}

The present section concerns the assessment of the active flow control approach applied to a swept wing in transonic conditions. As for the $2 \mathrm{D}$ test case presented in the previous section, different test cases are investigated, with a combination of angles of attack and blowing momentum of the control devices.

The following angles of attack will be presented for the three-dimensional test case:

- $\alpha=2.00^{\circ}$ without blowing. Hereafter "low angle of attack, no TED"

- $\alpha=2.00^{\circ}$ blowing with $\operatorname{MFR}_{T E D}=49.4 \mathrm{~g} / \mathrm{s}$. Hereafter "low angle of attack, low momentum"

- $\alpha=2.00^{\circ}$ blowing with $\operatorname{MFR}_{T E D}=123.2 \mathrm{~g} / \mathrm{s}$. Hereafter "low angle of attack, high momentum"

- $\alpha=3.55^{\circ}$ without blowing. Hereafter "high angle of attack, no TED" 
- $\alpha=3.55^{\circ}$ blowing with $\operatorname{MFR}_{T E D}=49.4 \mathrm{~g} / \mathrm{s}$. Hereafter "high angle of attack, low momentum"

- $\alpha=3.55^{\circ}$ blowing with $\mathrm{MFR}_{T E D}=123.2 \mathrm{~g} / \mathrm{s}$. Hereafter "high angle of attack, high momentum

The approach is based on continuous blowing through a span-wise slot on the lower surface in the vicinity of the trailing edge, employed to modify the span-wise aerodynamic loading in order to increase the lift-to-drag ratio for a rigid wing. As in the previous case, the fluidic trailing edge device is developed and demonstrated for separation control to increase the buffet margin at transonic conditions by increasing the rear-loading of the wing.

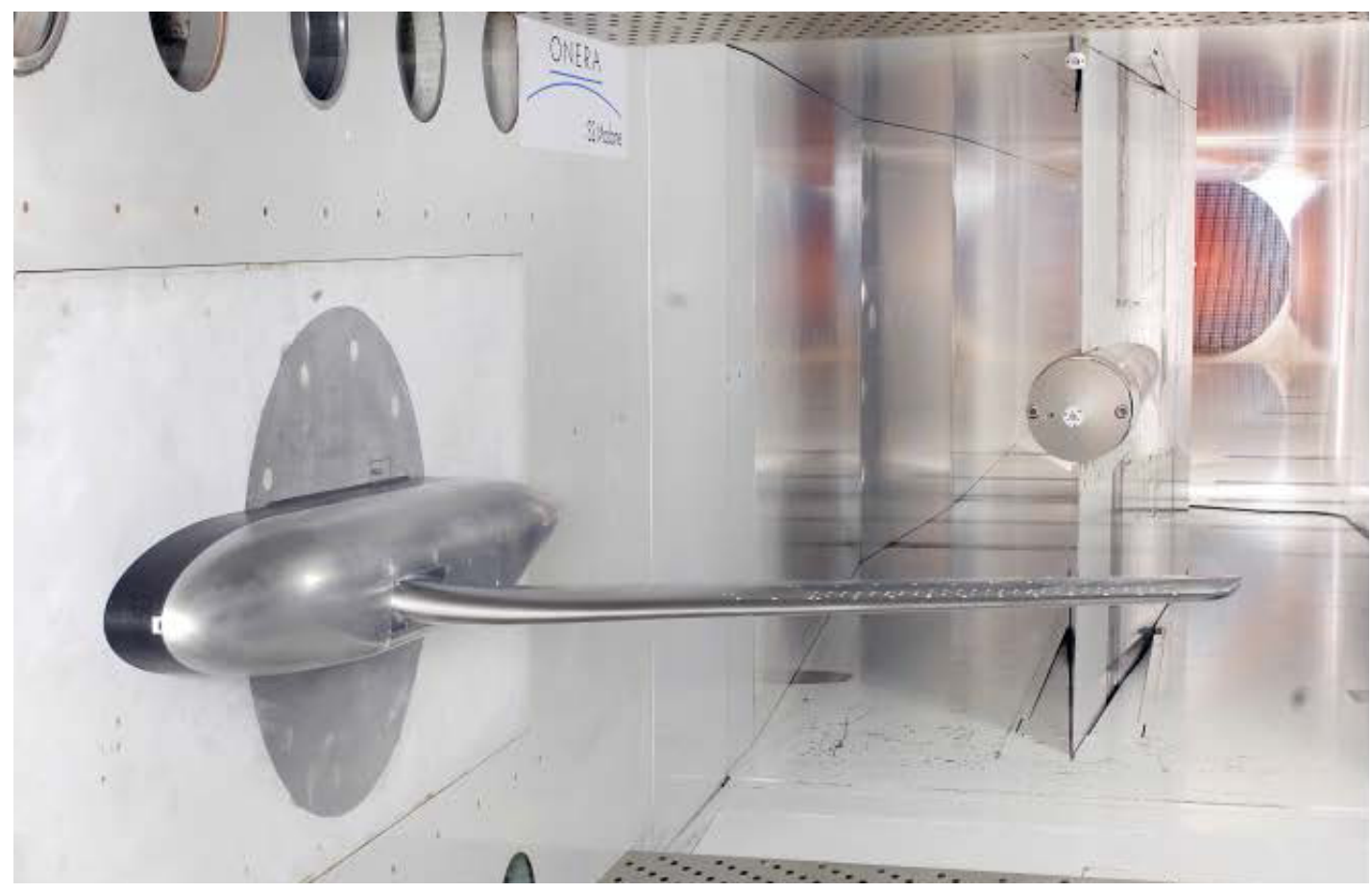

Figure 8: 3D model in the ONERA S2MA wind tunnel.

RANS approaches are used: each partner was free to use the best practice of its own code in terms of numerical method or turbulence model for the simulations. The test cases are selected from an available experimental data base obtained in the S2MA wind tunnel of ONERA. The comparison of numerical and experimental data concerns sectional pressure distributions on the wing and force coefficients for the entire configuration. Subsequently, lift curves are computed for the fluidic TED configurations at the design Mach number as a function of the jet momentum coefficient. The aerodynamic efficiency is determined in terms of the lift-to-drag ratio and compared to that of the baseline configuration. The half-model geometry consists of a wing, a fuselage and a peniche (see figure 8). The wing cross-section geometry is based on the OAT15A aerofoil.

The fluidic TED is equipped with a slot, the jet exit flow being normal to the lower surface. Its geometry is similar to the one used in the VZLU wind tunnel tests described above and has a slot width of $0.5 \mathrm{~mm}$. The chord-wise position of the slot center is at $x / c=95 \%$, while the span-wise length is $490 \mathrm{~mm}$. The slot is thus located between $45 \%$ and $85 \%$ of wing span. The design of the plenum that supplies the slot with air is based on the TED design for VZLU tests.

The mesh provided by ONERA consists of 249 blocks and 16.86 million grid points, including a description of the whole cavity of the TED and, contrarily to the 2D case in the previous section, is the same for all partners. A set of deformed grids were provided, taking into the account the static deformation of the wind-tunnel model. The boundary condition used for the jet computation can be either based on the specification of the total pressure, total temperature and velocity direction or on the direct specification of the mass flow rate though the control device. The actual values of pressure and 

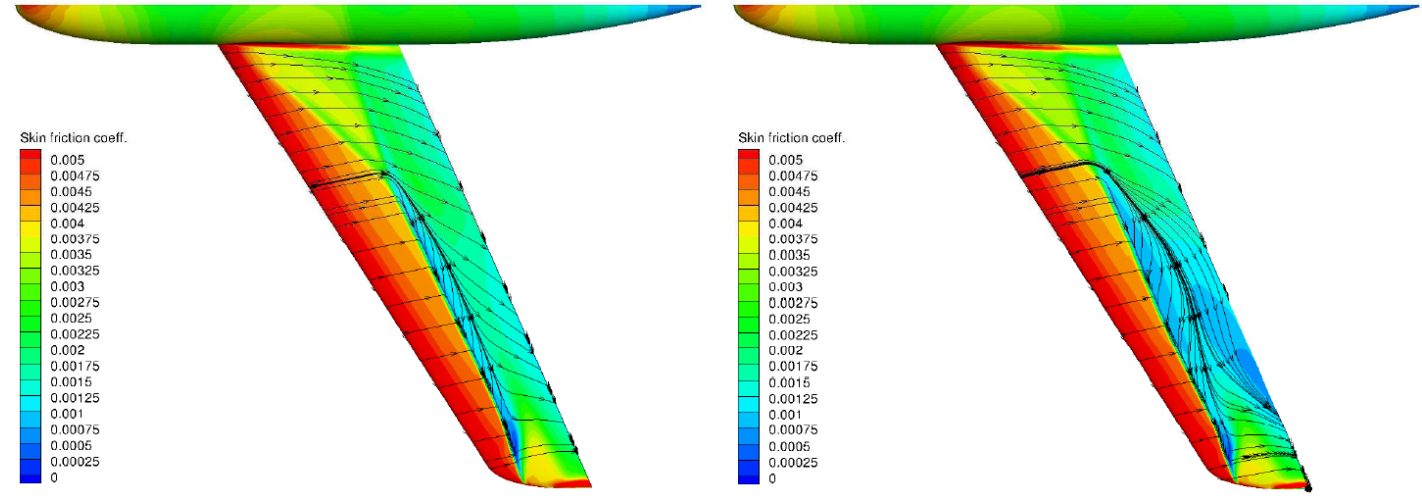

Figure 9: Skin friction on the 3D model from NLR: low (left) and high (right) angle of attack, no control.

temperature are prescribed in terms of the total pressure ratio and need to be adjusted in order to obtain the correct mass flow rate. NLR and TsAGI performed the simulations using this boundary condition, while ONERA used the second method.

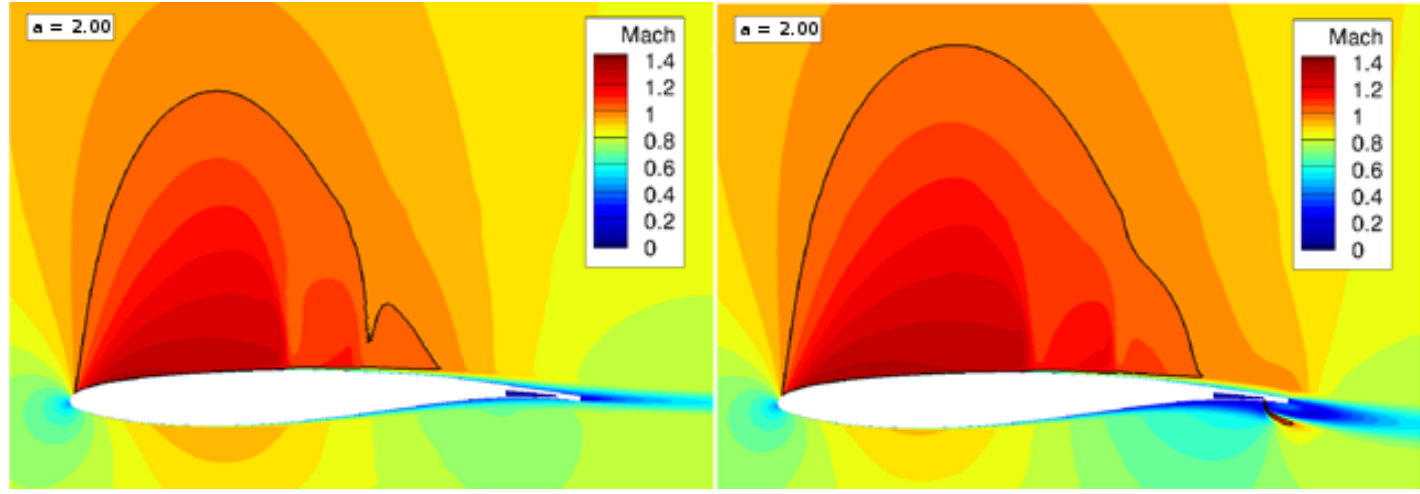

Figure 10: Mach field at $\mathrm{y} / \mathrm{b}=55.0 \%$ for the cases at low angle of attack, without (left) and with (right) flow control.

Figure 9 shows a typical flow solution, obtained by NLR using the baseline EARSM k- $\omega$ model at low (left side) and high (right side) angle of attack, indicating the flow pattern on the upper surface of the wing in terms of limiting stream-lines and illustrates the existence of a large separation bubble downstream of the shock location. When considering a lower angle of attack, the separated zone has a limited extension. As it can be seen in figure 10, showing the Mach distribution on a slice at $\mathrm{y} / \mathrm{b}=$ $55.0 \%$ of the span, the shock has a more downstream position. When the TED is active, the shock moves even further downstream, increasing the value of the total lift coefficient.

A comparison between the pressure coefficient distribution is presented in figure 11 for the uncontrolled cases: the agreement between the partners when using the same turbulence model is excellent, indicating that the failure to correctly reproduce the pressure distribution on the profile should not be sought in a specific code, but could be the consequence of the approach: RANS simulations, wall corrections, model deformations, and so on. On the same plot, the green line indicates a result obtained with the SA turbulence model: one can see that the comparison with the experimental data yields a better agreement when the angle of attack is small (left of the figure). However, when comparing the configurations at high angle of attack, the SA turbulence model predicts a shock position too far downstream, and the k- $\omega$ SST turbulence model yields a better agreement (right of the figure).

Figure 12 shows the pressure distribution for the span-wise location at $\mathrm{y} / \mathrm{b}=72.5 \%$ obtained for the two cases at low angle of attack, with and without control. The first case, on the left side of the image, presents the results for the case without TED and indicates that taking into account the 

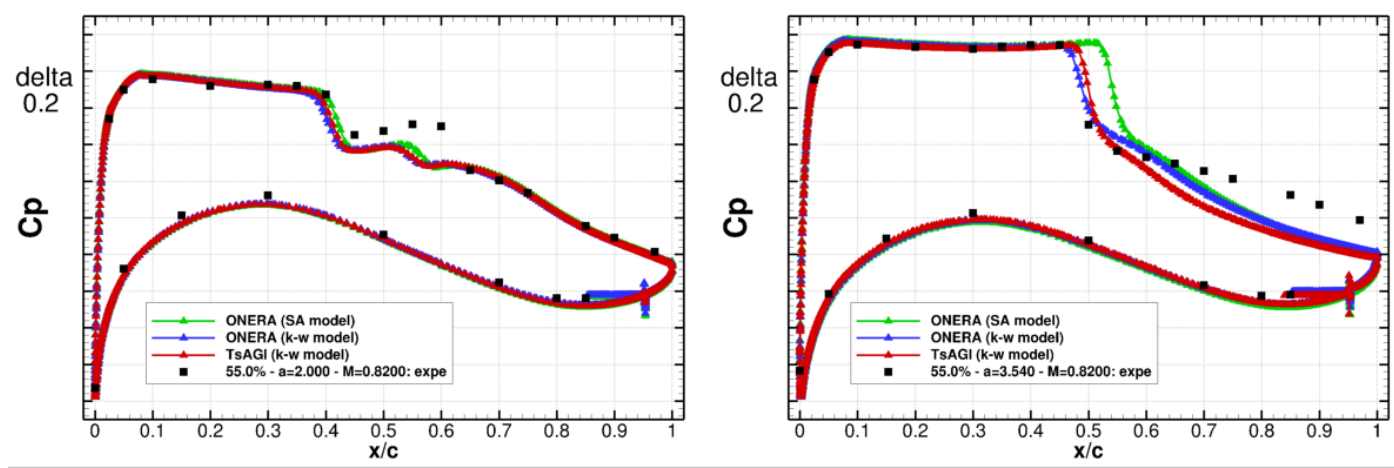

Figure 11: Pressure coefficient at $\mathrm{y} / \mathrm{b}=55.0 \%$ : low (left) and high (right) angle of attack, no control.

mesh deformation barely impacts the pressure distribution downstream of the shock. The shock wave however moves upstream when taking into the account the mesh deformation, yielding a less favourable comparison.


Figure 12: Pressure coefficient at $\mathrm{y} / \mathrm{b}=72.5 \%$ : low angle of attack, uncontrolled configuration (left), and case with flow control (right).

The case at low angle of attack, high momentum through the TED, is presented on the right side of figure 12. The TED directly impacts the pressure distribution of this wing section: the shock wave moves downstream with increasing momentum of the TED. Consequently, the shock-induced separation decreases its size thanks to the control device. The pressure in the trailing edge region is also decreased, inducing higher wing load and pitching moment. In this configuration the wing deformation is the most important, and a more favourable comparison with the experimental data can be observed when taking into the account the bending and twisting of the wing. Contrarily to the undeformed geometry, the secondary expansion seen in the pressure distribution downstream of the shock wave is correctly reproduced by the numerical simulations.

In conclusion, the selected simulation approach for the characterization of 3D buffet is based on RANS calculations in combination with different turbulence models. Assessment of numerical results in a comparison with experimental data obtained in the S2MA wind tunnel of ONERA show a good agreement in terms of wing sectional pressure distributions and aerodynamic force coefficients for the entire configuration. The evaluation of the fluidic TED as a trailing edge variable camber device shows an aerodynamic efficiency for a wing-body configuration in terms of an increase of the lift-to-drag ratio along the entire lift curve.

The benefit offered by the fluidic TED scales with the mass flow rate. Although a satisfying agreement is generally achieved with the original conditions, some more challenging cases exist, where the numerical simulation is not able to accurately reproduce the pressure distribution observed in the experiments at both span-wise locations. In most cases at low angle of attack, the modified conditions provided by 
ONERA to take into account the wind tunnel correction and the model deformations do not improve the agreement with the experimental data. The reason for this could be sought either on the wind tunnel corrections, or on the RANS approach, which fails to correctly predict the flow field on the wing surface. However, satisfying agreement is often already achieved when using the non corrected aerodynamic conditions. This indicates that if the purpose is to achieve an accurate prediction of the flow-field for each case, a more detailed study should be considered. However, if the goal of the study is to evaluate the effectiveness of the TED, a preliminary study can be considered sufficient, without considering wing deformation and wind tunnel correction.

In order to assess the effect of the fluidic TED on the aerodynamic performance at cruise conditions, the drag behaviour is computed for the clean and fluidic TED configurations at the design Mach number. The design point is defined as $C_{L}=0.5$. The clean configuration serves as a reference. The mass flow rate is varied for the TED configurations in order to identify the effect of the mass flow rate on the aerodynamic performance of the wing-body configuration. Figure 13 depicts the drag at two angles of attack for the clean and fluidic TED configurations. The mass flow rate is increased by considering the cases with higher momentum of the TED. The plot shows a reduction of the drag at a given value of the lift coefficient, which implies an enhancement of the aerodynamic efficiency.

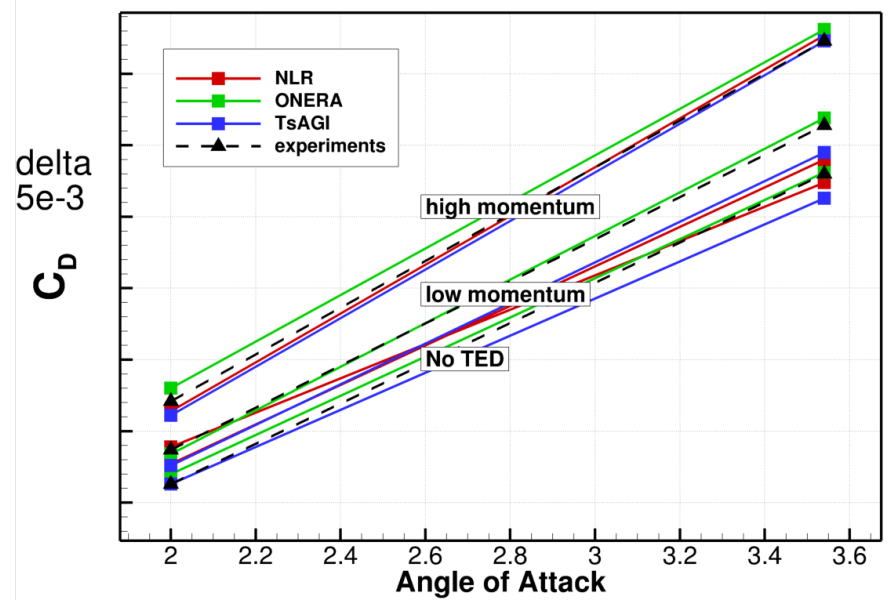

Figure 13: Comparison of drag coefficients between partner's results for all cases.

Finally, it is relevant to remark that relatively small drag increase is found for relatively small values of the jet momentum coefficient. These observations underline the multi-functional use of the fluidic TED for separation control and overall cruise performance enhancement. The fluidic TED seems a promising concept for future wings based on composite structures that feature larger flexibility than conventional wings. The aero-elastic deformations that originate from the variation in fuel load during the cruise segment result in aerodynamic performance degradation. The fluidic TED is capable to increase the local aerodynamic loading by increasing the rear-loading at the wing section. Therefore, the fluidic TED can be exploited to alter/restore the aerodynamic load distribution and act on the induced drag to limit the aerodynamic performance degradation.

\section{CONCLUSIONS}

The purpose of this article was to present the main results of the investigation on the potential of active pneumatic trailing edge flow control concepts. This was done in the framework of the European project AFLoNext by application of numerical simulations together with targeted simple experiments to understand the potential aerodynamic performance benefits achievable together with design parameters.

A benchmark between different partners involved in the project has been considered. Overall, the results of both $2 \mathrm{D}$ and $3 \mathrm{D}$ benchmarks show a good agreement between parters. An improvement of the lift-to-drag ratio for the considered lift coefficient range which is representative for the entire cruise 
segment of the flight, confirming the conclusion of the AVERT program. The benefit of the fluidic TED is relative large even for small values of the jet momentum coefficient. Most importantly, the results presented indicate that the numerical simulations compare favourably to the experimental results. Thus, CFD can be used as a reliable tool to predict the behaviour of the flow in the presence of control devices.

In terms of perspectives, a great advantage of the TED when compared to other classical control devices such as vortex generators is that the TED can be used as a pulsed device, either in an open or a closed loop approach. The efficiency of a closed-loop approach using pulsed TED depends on the choice of the control law, which is not trivial, and could be the subject of future studies, who will prove the ability of a pulsed device to obtain the same beneficial effect as the classical continuously-blowing device, but using less mass flow rate.

\section{Acknowledgements}

The work described in this paper and the research leading to these results has received funding from the European Commission Seventh Framework Programme FP7/2007-2013, under grant agreement $\mathrm{n}^{\circ} 604013$, AFLoNext project. The authors are also grateful to Lars Tysell from FOI for providing some of the results for this study, and to Mark Nichols from BAE Systems for managing the work package.

\section{REFERENCES}

[1] Marongiu C., Catalano P., Amato M., and Iaccarino G. U-zen: A computational tool solving URANS equations for industrial unsteady applications. In 34th AIAA Fluid Dynamics Conference and Exhibit, page 2345, 2004.

[2] Van Dam C.P., Yen D.T., and Vijgen P.M. Gurney flap experiments on airfoil and wings. Journal of aircraft, 36(2):484-486, 1999.

[3] Caruana D., Mignosi A., Corrège M., Le Pourhiet A., and Rodde A.M. Buffet and buffeting control in transonic flow. Aerospace science and technology, 9(7):605-616, 2005.

[4] Quagliarella D. and Vicini A. Gas for aerodynamic shape design. I: General issues, shape parameterization problems and hybridization techniques. Lecture series-van Kareman Institute for fluid dynamics, 7:O1-O24, 2000.

[5] Schwamborn D., Gerhold T., and Heinrich R. The DLR TAU-code: recent applications in research and industry. In ECCOMAS CFD 2006: Proceedings of the European Conference on Computational Fluid Dynamics, Egmond aan Zee, The Netherlands, September 5-8, 2006. Delft University of Technology; European Community on Computational Methods in Applied Sciences (ECCOMAS), 2006.

[6] Jeffrey David., Zhang Xin., and Hurst David W. Aerodynamics of gurney flaps on a single-element high-lift wing. Journal of Aircraft, 37(2):295-301, 2000.

[7] D. Dolgopyat. On the feasibility of hinge-less flow control manoeuvring system. Tel Aviv University, Israel, Master Thesis, 2014.

[8] Dvorak F.A. and Choi D.H. Analysis of circulation-controlled airfoils in transonic flow. Journal of Aircraft, 20(4):331-337, 1983.

[9] Arwatz G., Fono I., and Seifert A. Suction and Oscillatory Blowing actuator. In IUTAM Symposium on Flow Control and MEMS, pages 33-44, 2008.

[10] Browaeys G., Collin E., Deniau H., and Bonnet J.P. Numerical study of closed loop control of transonic buffet by means of fluidic trailing edge device. In CEAS/KATnet (Key Advances Technologies) Conference 2009, 2009. 
[11] Browaeys G., Deniau H., Collin E., and Bonnet J.P. Pneumatic control strategies for transonic buffet. a numerical approach. In 7th International Symposium on Engineering Turbulence Modeling and Measurement (ETMM7), Limassol, June 4-6, 2008., pages pp-805, 2008.

[12] Liebeck Robert H. Design of subsonic airfoils for high lift. Journal of aircraft, 15(9):547-561, 1978.

[13] Dandois J., Lepage A., Dor J.B., Molton P., Ternoy F., Geeraert A., Brunet V., and Coustols É. Open and closed-loop control of transonic buffet on 3d turbulent wings using fluidic devices. Comptes Rendus Mécanique, 342(6):425-436, 2014.

[14] Dandois J., Gleyzes C., Dor J-B., Ternoy F, and Coustols E. Report on the VZLU wind tunnel test analysis and report on the 3D RANS and URANS computations of mechanical/fluidic VGs and fluidic TEDs. AVERT Deliverables D1.3-3 \& D1.3-8, June 2010.

[15] Dandois J., Dor J.-B., Lepage A., Brunet V., Eglinger E., Ternoy F., and Coustols E. AVERT: ONERA S2MA wind tunnel test report. AVERT Deliverable D4.3-4, June 2010.

[16] Dandois J., Molton P., Lepage A., Geeraert A., Brunet V., Dor J-B., and Coustols E. Buffet characterisation and control for turbulent wings. Aerospace Lab Journal, 6, 2013.

[17] Hellsten Antti K. New advanced kw turbulence model for high-lift aerodynamics. AIAA journal, 43(9):1857-1869, 2005.

[18] Richter Kai. and Rosemann Henning. Steady aerodynamics of miniature trailing-edge devices in transonic flows. Journal of Aircraft, 49(3):898-910, 2012.

[19] Cambier L., Heib S., and Plot S. The ONERA elsA CFD software: input from research and feedback from industry. Mechanics and Industry, 14:159-174, 12013.

[20] Heeb N., Gutmark E., Liu J., and Kailasanath K. Fluidically enhanced chevrons for supersonic jet noise reduction. AIAA Journal, 52(4):799-809, 2014.

[21] Eliasson P. EDGE, a Navier-Stokes solver for unstructured grids. Proceedings to Finite Volumes for Complex Applications III, pages 527-534, 2002.

[22] Molton P., Dandois J., Lepage A., Brunet V., and Bur R. Control of buffet phenomenon on a transonic swept wing. AIAA journal, 51(4):761-772, 2013.

[23] Wallin S. and Johansson A.V. An explicit algebraic reynolds stress model for incompressible and compressible turbulent flows. Journal of Fluid Mechanics, 403:89-132, 2000.

[24] P. Vrchota, A. Prachar, P. Hospodar, D. Dolgopyat, and A. Seifert. Development of simplified boundary condition of saob actuator based on high-fidelity cfd simulations. In Proceedings of the 52nd 3AF International Conference on Applied Aerodynamics, Lyon - France, 2017. 